\title{
Perfil de morbilidad por enfermedades infecciosas de las comunidades indígenas pertenecientes al Departamento de Casanare, Colombia.
}

\author{
Martha Leonor Castillo Bohórquez MSc. ${ }^{1}$, Ana lucia oliveros Rozo MSc. ${ }^{1}$, \\ Ana IsabelMora Bautista ${ }^{1}$ Mag., Jennifer Andrea Contador Alonso ${ }^{2}$, Lesley \\ Andrea Cortés Jaimes², Jeny Carolina Mora Acero ${ }^{2}$
}

\footnotetext{
1. Docentes investigadoras semillero ERITRON Universidad Colegio Mayor de Cundinamarca.

2. Estudiantes del programa de Bacteriología y Laboratorio Clínico de la Universidad

Colegio Mayor de Cundinamarca.
}

Correspondencia: mlcastillo@unicolmayor.edu.co

Recibido: 07/06/2012 Aceptado: 18/10/2012

\section{Resumen}

El propósito de esta investigación fue establecer el perfil de morbilidad infecciosa en la población indígena ubicada en el departamento de Casanare durante los años 2010 a marzo de 2012. Se utilizaron datos registrados por las instituciones encargadas de su atención y los recolectados por la Secretaría de Salud de Yopal, clasificándolos por resguardo: Caño Mochuelo, Chaparral Barro Negro y Orocué. Los resultados mostraron que las enfermedades de tipo infeccioso prevalentes en las comunidades indígenas estudiadas son: diarrea con 702 casos, infección de vías respiratorias 570 casos y parasitismo intestinal 187 casos.

Palabras clave: perfil de morbilidad, población indígena, enfermedad infecciosa, Casanare (Colombia).

\section{Infectious disease morbidity profile of indigenous communities from department of Casanare (Colombia)}

\begin{abstract}
The purpose of this research was to establish the infectious disease profile of the indigenous population located in the Casanare department during the years 2010, 2011 and March 2012. We used data collected for the institutions in charge of the indigenous health care and gathered for the Yopal Department of health (Casanare, Colombia) classified by indigenous reservation: Caño Mochuelo, Chaparral Barro Negro and Orocué. The results showed that prevalent infectious diseases in the indigenous community are diarrhea, respiratory tract infection and intestinal parasitism with 702, 570 and 187 cases respectively.
\end{abstract}

Key words: morbidity profile, indigenous population, infectious disease, Casanare (Colombia). 


\section{Introducción}

Colombia, ubicada al noroccidente de Sur América, es un país rico y complejo por su diversidad biológica y su espacio sociocultural caracterizado por la diversidad de comunidades indígenas (1). Según el censo general 2005, en Colombia residen 87 pueblos indígenas identificados plenamente; estos grupos viven en diferentes lugares y son muy diversas las estrategias que han adoptado para vivir (2). Se encuentran ubicados en las regiones naturales como la selva, las sabanas de la Orinoquia, los Andes colombianos, en los valles interandinos y en la planicie del Caribe (3).

El departamento de Casanare representa un porcentaje significativo de dichas etnias. La ubicación actual de los indígenas de este lugar está concentrada en cuatro municipios: Hato Corozal y Paz de Ariporo con el resguardo Caño Mochuelo, Orocué conformado por los resguardos de El Duya, San Juanito, El Consejo, Macucuana, Paravare, El Medano, Saladillo y El Suspiro y, finalmente Sácama en el que se encuentra el resguardo Chaparral Barro Negro.

El estudio se realizó en los pueblos indígenas; Amorúa, Kuiba, Sikuani, Masiguare, Tsiripu dentro del resguardo de Caño Mochuelo; Sáliba en los resguardos del municipio de Orocué y U'wa en el resguardo de Chaparral Barro Negro. Se recopilaron datos de diagnóstico clínico de los miembros de las poblaciones en mención suministrados por las entidades gubernamentales encargadas. Los datos obtenidos se clasificaron por género, edad y resguardo indígena.

Ésta investigación tuvo por objeto establecer el perfil de morbilidad infecciosa de dichas comunidades y reconocer las principales enfermedades de este tipo que afectan a la población indígena de Casanare. La investigación surgió a partir de la falta de datos indicadores del estado de salud actual de los indígenas para enriquecer la estadística epidemiológica de estas comunidades. Es necesario mencionar que la falta de datos obedece a razones como el difícil acceso a los resguardos, las creencias de la etnia, la falta de recursos y en ocasiones, las condiciones ambientales.

\section{Materiales y métodos}

Población: población indígena nativa de las familias lingüísticas Guahibo, con sus grupos indígenas Amorúa, Kuiba, Sikuani, Masiguare y Tsiripu; familia Sáliba con el grupo indígena Sáliba y de la familia Chibcha el grupo U'wa, ubicadas en los resguardos Caño Mochuelo, Orocué y Chaparral Barro Negro respectivamente.

Recolección de los datos: los datos fueron tomados del archivo estadístico de morbilidad de los grupos indígenas pertenecientes a los resguardos de Caño Mochuelo, Chaparral Barro Negro y Orocué ubicados en Casanare suministrados por el Hospital de Yopal E.S.E. y las I.P.S. encargadas de ellos en el periodo del año 2010 a marzo de 2012.

Análisis estadístico: los datos fueron clasificados por resguardo indígena: Caño Mochuelo, Chaparral Barro Negro y Orocué, posteriormente los diagnósticos fueron discriminados por enfermedad infecciosa. Se obtuvieron los porcentajes según la población afectada.

\section{Resguardo Caño Mochuelo}

Según la brigada de salud realizada por el Hospital de Yopal E.S.E. en marzo de 2012, la población indígena del resguardo Caño Mochuelo corresponde a 1458 personas de las cuales 865 son del género femenino y 593 del género masculino (4).

Morbilidad sentida. Los datos encontrados de morbilidad sentida de cada comunidad del resguardo Caño Mochuelo fueron resumidos en la tabla 1. En ésta se observan tres parámetros: el nombre cotidiano que es con el que comúnmente se conoce la enfermedad, el nombre en lengua indígena y el nombre facultativo, que es el mismo nombre científico de cada patología. Cabe resaltar que estos datos son epidemiológicos culturales, es decir lo 
que los indígenas por sus creencias o conocimientos creen padecer.

Tabla 1. Morbilidad sentida etnias del resguardo Caño Mochuelo.

\begin{tabular}{lll}
\multicolumn{1}{c}{$\begin{array}{c}\text { Nombre } \\
\text { cotidiano }\end{array}$} & \multicolumn{1}{c}{$\begin{array}{c}\text { Nombre en } \\
\text { lengua indígena }\end{array}$} & \multicolumn{1}{c}{$\begin{array}{c}\text { Nombre } \\
\text { facultativo }\end{array}$} \\
Gripa & Buco domae & Gripe \\
Diarrea & Sulia & Diarrea \\
Tuberculosis & Maneni & Tuberculosis \\
Suin & Tsatsani & Candidiasis \\
Marranas & Tekerto & Esporotricosis \\
Tísico & - - & Tuberculosis \\
Dolor de oído & - - & Otitis \\
Sabańones & Xáa ichi & Tińa pedís \\
Paludismo & Sódi nochi & Malaria \\
Chúcharos & - - & Furunculosis \\
Carranchil & - - - & Escabiosis \\
Tos & Buju & - - - \\
\hline
\end{tabular}

Fuente: Hospital de Yopal E.S.E.

Morbilidad. La morbilidad es la confirmada por el profesional competente, en la tabla 2 se establecen las cuatro causas de origen infeccioso más frecuentes para el Resguardo Caño Mochuelo a marzo de 2012.

Tabla 2. Morbilidad Resguardo Caño Mochuelo a marzo de 2012.

\begin{tabular}{|lcc|}
\hline \multicolumn{1}{|c}{ Diagnóstico } & $\begin{array}{c}\text { Número de } \\
\text { personas }\end{array}$ & Porcentaje \\
\hline Parasitosis intestinal. & 187 & $58 \%$ \\
\hline $\begin{array}{l}\text { Infección aguda de las vías } \\
\text { respiratorias superiores. }\end{array}$ & 78 & $24 \%$ \\
\hline $\begin{array}{l}\text { Rinofaringitis aguda } \\
\text { (resfriado común) }\end{array}$ & 32 & $10 \%$ \\
\hline $\begin{array}{l}\text { Diarrea y gastroenteritis de } \\
\text { presunto origen infeccioso }\end{array}$ & 27 & $8 \%$ \\
\hline Total & 324 & $100 \%$ \\
\hline
\end{tabular}

Fuente: Hospital de Yopal E.S.E.

\section{Resguardo Chaparral Barro Negro}

De acuerdo con los datos aportados por la Secretaría de Salud de Casanare, en el resguardo Chaparral Barro Negro se registraron 460 personas, de las cuales el $49 \%$ son del género femenino y el $51 \%$ restante del género masculino (6).

Morbilidad sentida. Al igual que en el resguardo Caño Mochuelo, en Chaparral Barro Negro también se presenta la gripa como morbilidad sentida además de la parasitosis, tabla 3.

Tabla 3. Morbilidad sentida etnia $U^{\prime} w a$.

\begin{tabular}{|c|c|c|}
\hline \multicolumn{3}{|c|}{ ETNIA U' WA } \\
\hline $\begin{array}{l}\text { Nombre } \\
\text { cotidiano }\end{array}$ & $\begin{array}{c}\text { Nombre en } \\
\text { lengua nativa }\end{array}$ & $\begin{array}{c}\text { Nombre } \\
\text { facultativo }\end{array}$ \\
\hline Gripa & Okuará & Rinofaringitis \\
\hline & Wishiriara & Diarrea \\
\hline $\begin{array}{l}\text { Dolor del cuer- } \\
\text { po, "Dengue" }\end{array}$ & Okiará & -- \\
\hline Parásitos & Sianá & $\ldots$ \\
\hline $\begin{array}{l}\text { Hongos en } \\
\text { la piel }\end{array}$ & Owasa & Dermatomicosis \\
\hline "Asma" & Tanasi & Neumonía \\
\hline
\end{tabular}

Fuente: Hospital de Yopal E.S.E.

Morbilidad. Según los RIPS (Registro Individual de Prestación de servicios de Salud) de los servicios de consulta externa, hospitalización y urgencias (6) de la EPS Capresoca encargada de la atención en salud de la población indígena de Casanare, las principales causas de morbilidad en el ańo 2010 fueron:

(i) Servicio de consulta externa: neumonía bacteriana, amigdalitis, otitis media supurativa, parasitosis intestinal.

(ii) Servicio de hospitalización: insuficiencia respiratoria aguda, neumonía, bronconeumonía, neumonía bacteriana, absceso cutáneo.

(iii) Servicio de Urgencias: micosis superficial, varicela, bronconeumonía, infecciones locales piel y tejido subcutáneo, infección viral, enfermedad parasitaria. 
Se encontró que las comunidades habitan viviendas construidas por techos de palmas, paredes en tablas y piso en tierra. Los factores climáticos influyen en la transmisión de enfermedades y los resguardos no cuentan con agua potable, electricidad, ni lugar de descarte de desechos (6).

\section{Resguardos de Orocué}

La población indígena de Orocué se compone de 1535 personas, de las cuales el $49.9 \%$ son mujeres y $50.1 \%$ son hombres (8).

Morbilidad. En la tabla 4 se incluyen las tres primeras causas de morbilidad de los indígenas del resguardo Orocué, se puede observar que predomina la diarrea afectando a 675 personas con un porcentaje de $51 \%$, es decir, la padece más de la mitad de la población, en segundo y tercer lugar se encuentran las infecciones respiratorias y urinarias con un $37 \%$ y $12 \%$ de prevalencia respectivamente.

Tabla 4. Morbilidad de la población indígena de Orocué en el año 2011.

\begin{tabular}{l|l|l|}
\hline \multicolumn{1}{|c|}{ Diagnóstico } & \multicolumn{1}{c|}{$\begin{array}{c}\text { Número de } \\
\text { personas }\end{array}$} & \multicolumn{1}{c|}{ Porcentaje } \\
\hline Diarrea & 675 & $51 \%$ \\
\hline $\begin{array}{l}\text { Infecciones } \\
\text { respiratorias }\end{array}$ & 492 & $37 \%$ \\
\hline $\begin{array}{l}\text { Infecciones } \\
\text { Urinarias }\end{array}$ & 153 & $12 \%$ \\
\hline TOTAL & $\mathbf{1 3 2 0}$ & $100 \%$ \\
\hline
\end{tabular}

Fuente: Asociación de Autoridades Indígenas Sáliba de Orocué Casanare. 2011

\section{Discusión}

En el presente trabajo se investigó la morbilidad infecciosa en la población indígena del Departamento de Casanare, Colombia. Se hizo una recolección de datos diagnósticos clínicos de la etnia y se clasificaron por edad, género y resguardo: Caño Mochuelo, Chaparral Barro Negro y Orocué, se analizó la morbilidad presentada en los indígenas de dichos resguardos teniendo en cuenta la morbilidad sentida.
De acuerdo con los resultados de morbilidad sentida encontrados en este estudio, la población de los resguardos Caño Mochuelo y Chaparral Barro Negro informan, desde su perspectiva, el padecimiento de enfermedades como la gripa y la diarrea; cabe resaltar que dichas patologias son diagnosticadas de acuerdo a los sintomas de los encuestados, en efecto, para el grupo investigador, esto produce una generalización arriesgada de las enfermedades, más aun teniendo en cuenta la ausencia de fundamentación cientifica, pues los síntomas mencionados por los indígenas no necesariamente indican que esa sea la enfermedad padecida. De esta forma, para tener un diagnóstico sería necesario un chequeo médico y pruebas se laboratorio que puedan conducir al dictamen.

Con respecto a los datos epidemiológicos recolectados, se evidenció la diarrea como enfermedad prevalente en los resguardos de Orocué con 51\%, la parasitosis intestinal predominó en el resguardo Caño Mochuelo afectando a toda la población pero en mayor proporción a los niños de 5 a 14 años con una prevalencia de $57 \%$. Las enfermedades parasitarias representan una de las principales causas de enfermedad, se presentan en todas las edades pero más en los niños(9).

Por último, la infección respiratoria se presentó en los dos resguardos con un porcentaje de 37 y $24 \%$ respectivamente. Esto puede ser la consecuencia de la carencia de mecanismos de acceso a la medicina facultativa por parte de las comunidades indígenas. De acuerdo con el informe de "Atención diferencial para pacientes de comunidad indígena" del Plan de atención Básica Indígena del Hospital de Yopal E.S.E., esta población debe recorrer grandes distancias para acceder a la atención médica y a los medicamentos, de ahí que los miembros de la comunidad acudan en primera instancia a la medicina tradicional que se mantiene vigente al interior de los resguardos (10).

También influyen las costumbres propias de los indígenas como caminar sin el calzado adecuado, beber agua sin tratamiento y cocinar sin lavar 
rigurosamente los alimentos, así lo indica el documento "Perfil epidemiológico resguardo indígena Chaparral Barro Negro", en éste se informa que la comunidad indígena ubicada en este resguardo tiene acceso al agua a través de un río o caño, un pozo y un aljibe, en donde el $92 \%$ de esta población consume el agua cruda y un $8 \%$ consume el agua hervida (6), siendo esta una posible causa de infecciones parasitarias y/o de enfermedad diarreica aguda.

Datos concordantes con el estudio de autoría de Rodriguez, M (11), titulado: "Determinantes de la mortalidad y la prevalencia de enfermedades diarreicas agudas en menores de cinco años en Colombia" publicado en el 2012, en el que se afirma que la prevalencia de la enfermedad diarreica aguda es de 19,1\% entre los nińos cuyas familias obtienen el agua de pozos abiertos y de un porcentaje menor $(12,2 \%)$ para quienes la obtienen por tuberías (11). Es pertinente resaltar que el agua, conjuntamente con los alimentos contaminados es el principal vehículo para que este tipo de enfermedades llegue a las personas, de ahí la importancia de contar con agua potable (12).

Así mismo, la infección respiratoria aguda es también una enfermedad prevalente presentándose en los resguardos indígenas estudiados con porcentajes representativos de 37 y 58\%. Así se evidencia en el documento "Estado de salud infantil en las comunidades indígenas, afrocolombianas y aisladas en Colombia" de Nuñez, F et al (13), en el cual describen esta enfermedad como una de las que predomina en la población indígena, seguida por la enfermedad diarreica aguda (13).

Dentro de los riesgos para padecer EDA se encuentran: el estrato socioeconómico, el ingreso familiar, las condiciones higiénicas del hogar y de las personas, los hábitos de nutrición, la calidad de la alimentación y el peso al nacer (14). Tal como se observó en el desarrollo de la investigación, los hallazgos del estudio demostraron la prevalencia de parasitosis intestinal en la población indígena de
Casanare, estas comunidades dada su ubicación geográfica son más vulnerables a las parasitosis intestinales, afectadas principalmente por sus bajos ingresos, condiciones sanitarias deficientes y la carencia de servicios básicos de salud (15).

A su vez, se dificultó encontrar información detallada de enfermedades infecciosas, en los resguardos de Chaparral Barro Negro y los pertenecientes al municipio de Orocué, debido a que la sistematización de los datos recolectados, ya sea por brigadas o entidades prestadoras de salud, no especifica pertenencia étnica.

\section{Conclusiones}

Como enfermedades principales por resguardos, se encontró que la parasitosis intestinal prevalece en el resguardo Caño Mochuelo con un 58\% además de la infección respiratoria aguda en un $26 \%$. Esta última patología se manifiesta a su vez en los resguardos de Orocué con un 37\% y también se reportan casos de diarrea con un $51 \%$. El resguardo de Chaparral Barro Negro informa enfermedades como la neumonía, infección respiratoria agua y de tipo parasitario, aunque de estas no se registran cifras estadísticas que permitan analizar la prevalencia de las mismas.

Por último, se evidencia la falta de sistematización de datos diagnósticos por pertenencia étnica que permitan identificar las patologías de los pacientes indígenas.

\section{Referencias}

1. Arango R, Sánchez E. Los pueblos indígenas de Colombia en el umbral del nuevo milenio. Departamento Nacional de Planeación. Bogotá, Colombia, 2006.

2. Arango R, Sánchez E. Capítulo IV Los pueblos indígenas y su patrimonio cultural. Los pueblos indígenas de Colombia en el umbral del nuevo milenio. Departamento Nacional de Planeación. Bogotá, Colombia, 2006.

3. Dirección de censos y demografía. Colombia una nación multicultural, su diversidad étnica. Departamento Administrativo Nacional de Estadística (DANE). 2007.

4. Resultados de encuesta aplicada en brigada de salud realizada del 19 de marzo al 24 de marzo del 2012, en la población indígena de San José del Ariporo, del municipio de Paz de Ariporo por el Hospital de Yopal E.S.E. 
5. Plan de Atención Básica Indígena. Propuesta de modelo de atención en salud Resguardo de Caño Mochuelo. Yopal, Casanare. Hospital de Yopal E.S.E., 2007.

6. Perfil epidemiológico resguardo indígena Chaparral Barro Negro. Asociación De Autoridades Indígenas Sáliba de Orocué - Casanare. 2010.

7. Plan de Atención Básica Indígena. Propuesta de modelo de atención en salud núcleos indígenas del departamento de Casanare. Yopal, Casanare, Hospital de Yopal E.S.E., 2007.

8. Perfil epidemiológico intercultural del pueblo indígena sáliba. Asociación de Autoridades Indígenas Sáliba de Orocué - Casanare. 2011

9. Romero, R. Microbiología y Parasitología Humana. Mexico D.F.: Editorial Médica Panamericana; 2007.

10. Plan de atención Básica Indígena. Atención diferencial para pacientes de comunidad indígena. Hospital de Yopal E.S.E. 2007.
11. Rodríguez M. Determinantes de la mortalidad y la prevalencia de enfermedades diarreicas agudas en menores de cinco años en Colombia.. Economía \& Región, 2012; Vol. 6; 1: 5-26.

12. Gómez, H. Enfermedad diarreica aguda. Sociedad Colombiana de Pediatría [en línea]. Atlántico:; [Recuperado el 05 de 04 de 2012]. Disponible en: http://www.pediatriaatlantico.org/ articulos/enfermedad diarreica aguda.htm

13. Nuñez, F et al. Estado de salud infantil en las comunidades indígenas, afrocolombianas y aisladas en Colombia. Instituto de genética humana. Bogotá: Pontificia Universidad Javeriana; 2001.

14. Alba, L. Enfermedad diarreica aguda en adultos. En: R. R. Uribe. Guías de práctica clínica de las patologías gastrointestinales. Bogotá: Ediciones Médicas Latinoamericanas; 2003: 60-65.

15. Mora, L. Parasitosis intestinales y factores higienicos sanitarios asociados en individuos de localidades rurales del estado de Sucre. Kasmera. 2009:148-156. 